\title{
Failure of endoscopic management of ureteral strictures is due to ureteroscopic lithotripsy as a cause and longer length of stricture
}

\section{Teruaki Sugino}

Nagoya City University Graduate School of Medical Sciences

Kazumi Taguchi ( $\sim$ ktaguchi@med.nagoya-cu.ac.jp )

Nagoya City University Graduate School of Medical Sciences

https://orcid.org/0000-0002-3092-5114

Shuzo Hamamoto

Nagoya City University Graduate School of Medical Sciences

Tomoki Okada

Nagoya City University Graduate School of Medical Sciences

Masahiko Isogai

Nagoya City University Graduate School of Medical Sciences

\section{Yutaro Tanaka}

Nagoya City University Graduate School of Medical Sciences

Rei Unno

Nagoya City University Graduate School of Medical Sciences

Yasuhiro Fujii

Nagoya City University Graduate School of Medical Sciences

Takashi Hamakawa

Nagoya City University Graduate School of Medical Sciences

Ryosuke Ando

Nagoya City University Graduate School of Medical Sciences

Atsushi Okada

Nagoya City University Graduate School of Medical Sciences

Takahiro Yasui

Nagoya City University Graduate School of Medical Sciences

Research article

Keywords: ureteroscopy, ureteral stricture, impacted stones, hydronephrosis, prestenting

Posted Date: August 28th, 2020

DOl: https://doi.org/10.21203/rs.3.rs-63640/v1 
License: (c) (i) This work is licensed under a Creative Commons Attribution 4.0 International License. Read Full License 


\section{Abstract \\ Background}

Ureteroscopic lithotripsy is a common treatment for middle and lower ureteral stones. Although effective and minimally invasive, this technique might cause significant complications, including postoperative ureteral strictures, which occur in $1-4 \%$ of patients after ordinary ureteroscopic lithotripsy and in $7.8-$ $24 \%$ when lithotripsy is performed for impacted stones. The main purpose of the management of ureteral strictures is to improve hydronephrosis and protect renal function. However, factors that influence the success rate of the endoscopic management of US (e.g., the cause and length of stricture, the duration of hydronephrosis, surgical management technique, and the number of placed ureteral stents) are controversial. The aim of this study was to investigate factors determining the outcomes of endoscopic management for stone-related ureteral stricture.

\section{Methods}

This multi-center case series study was performed at one of the highest-volume centers and the affiliated institutions in Japan. Data of patients who underwent endoscopic surgery for ureteral stricture from January 2016 to March 2019 were retrospectively analyzed. Laser incision and/or balloon dilation were performed for management; single or double stents were placed at the end of the surgery. Treatment success was defined as improvement in hydronephrosis status.

\section{Results}

Nineteen patients were treated for stone-related ureteral stricture. Hydronephrosis successfully improved in 12 patients (63.2\%). All seven patients with failed endoscopic management had ureteroscopic lithotripsy-related stricture, whereas 3/12 (25.0\%) and 7/12 (58.3\%) patients with ureteroscopic lithotripsy-related stricture and impacted stone-related stricture, respectively, underwent successful endoscopic treatment $(P=0.004)$. Stricture length $>15 \mathrm{~mm}$ was observed in five patients $(71.4 \%)$ with failed management, two patients (16.6\%) with successful management $(P=0.046), 7 / 10$ patients $(70.0 \%)$ with ureteroscopic lithotripsy-related stricture, and $1 / 7$ patients $(14.3 \%)$ with impacted stone-related stricture $(P=0.049)$. Among patients with ureteroscopic lithotripsy-related stricture and impacted stonerelated stricture, both laser incision and balloon dilation were performed in 5/10 (50.0\%) and 2/7 (28.6\%) patients $(P=0.874)$ while double stents were placed in $5 / 10(50.0 \%)$ and $5 / 7(57.1 \%)$ patients $(P=0.874)$, respectively.

\section{Conclusions}


Ureteroscopic lithotripsy as a cause and stricture length $>15 \mathrm{~mm}$ could strongly affect the success rate of endoscopic management of ureteral stricture. In such cases, reconstructive management should be considered in the early stages.

\section{Background}

In recent years, the prevalence of ureteral stones has been consistently increasing in the world due to the effects of the increasing incidence of obesity and changes in dietary habits. ${ }^{1,2}$ Ureteroscopic lithotripsy (URSL) has become a common treatment for middle and lower ureteral stones. ${ }^{3,4}$ Although it is effective and minimally invasive, it could cause significant complications such as intraoperative ureteral injury, bleeding, infection, and postoperative ureteral strictures (US). ${ }^{5}$ US is reported to occur in $1-4 \%$ of patients after ordinary URSL; however, it occurs in $7.8-24 \%$ of patients when URSL is performed for impacted stones. ${ }^{6,7,8}$ Moreover, there are non-iatrogenic ureteric strictures such as those associated with impacted stones or chronic inflammatory disorders. ${ }^{9,10}$

The main purpose of the management of US is to improve hydronephrosis and protect renal function. ${ }^{11}$ Recently, a wide variety of therapeutic options has become available to urologists, such as endoscopic management and open/laparoscopic/robot assisted reconstruction. Laser incision (LI) and balloon dilation (BD) as endoscopic management techniques for benign US has been described in previous reports; Razdan et al. reported that these techniques had a success rate of $74 \%$ of 50 patients. ${ }^{9}$ Further, May et al. reported that $27.5 \%$ of 40 patients were successfully managed with endoscopic techniques. ${ }^{12}$ The factors that influence the success rate of the endoscopic management of US (e.g., the cause and length of stricture, the duration of hydronephrosis, surgical management technique, and the number of placed ureteral stents) are controversial.

To identify the factors associated with the successful endoscopic management of US, we retrospectively investigated 19 patients who underwent endoscopic management for benign US related to ureteral stones and/or their treatments.

\section{Methods}

\section{Patients}

The present study was approved by the Institutional Review Board of Nagoya City University Hospital. All patients provided informed consent for the use of their data.

Patients diagnosed with benign US between January 2016 and April 2020 were analyzed. These patients underwent $\mathrm{LI}$ and/or BD management and one or two ureteral stents or nephrostomy catheter were placed at the end of the surgery at Nagoya City University Hospital and two affiliated institutions. Patients with a solitary kidney, urinary diversion, poorly controlled diabetes, and those who were pregnant were excluded from this study. We obtained patients' demographics, such as sex, age, and BMI from the 
medical records. Additionally, the laterality, location, cause, and length of the US as well as the status of hydronephrosis were also captured. The US related to URSL was defined as follows: US without stone impaction, which was related to damage caused by the laser or access sheath during URSL. US related to an impacted stone was defined as follows: US with stone impaction which was observed during prior URSL.

\section{Surgical techniques}

All patients were placed under general anesthesia, and the operation was performed in the lithotomy position. Before treatment, the status of the stricture was confirmed using retrograde ureteropyelography. A semi-rigid ureteroscope (Olympus, Tokyo, Japan) was inserted and used to observe the stricture site. Then, we inserted a 0.035 -inch guide wire through the stricture site. As the LI procedure, we cut the mucosa and muscular layer of the stricture site using a $200 \mu \mathrm{m}$ fiber (Cyber Ho, Quanta system, Milan, Italy), including $5 \mathrm{~mm}$ before and after, until we could visualize the fat tissue outside of the ureter. The energy setting was $60 \mathrm{~W}$ and the incision was conducted using the 'Soft Tissues' mode. For the BD, we dilated the ureteral lumen up to $15 \mathrm{Fr}$ using a balloon catheter (URO MAX Ultra ${ }^{\mathrm{T}}$; Boston Scientific Japan, Tokyo, Japan). One or two double-J ureteral stents (4.7 Fr or 6.0 Fr, INLAY OPTIMA ${ }^{\mathrm{TM}}$, BD, Franklin Lakes, $\mathrm{NJ}$ and $4.8 \mathrm{Fr}$, Tria ${ }^{\mathrm{T}}$, Boston Scientific, Marlborough, MA) were placed into the ureter at the end of procedures.

\section{Follow-up}

Surgical parameters, including surgical time, postoperative hydronephrosis, and success or failure of treatment were analyzed. Furthermore, we evaluated laboratory tests. Success of treatment was defined as improvement in the status of hydronephrosis examined by either ultrasonography or computed tomography.

\section{Statistical analysis}

Non-normally distributed variables are expressed as medians (25\%, $75 \%$ interquartile range). Categorical variables are presented as frequencies (percentages). Data were analyzed using EZR for $R$ ( $R$ project 3.6.3). ${ }^{13}$ To compare the patients with failed or successful management and the factors associated with the cause of US, Fisher's exact test and Mann-Whitney U tests were used. To compare preoperative and postoperative renal function, the Wilcoxon signed-rank test was used. P-values $<0.05$ were defined as statistically significant.

\section{Results}

A total of 19 patients were identified as having undergone endoscopic treatment for US. The characteristics of the patients are summarized in Table 1. The stricture cause was identified as URSL in 10 patients $(52.6 \%)$, impacted stone in 7 patients (36.8\%), and chronic inflammatory disorders in 2 patients (10.5\%). The median length of the stricture was $10 \mathrm{~mm}$, and the median duration of hydronephrosis before surgery was 4.0 months. Preoperative hydronephrosis was Grade 1 in 3 patients 
(15.8\%), Grade 2 in 5 patients (26.3\%), Grade 3 in 5 patients (26.3\%) and Grade 4 in 6 patients (31.6\%). Prestenting before surgery was performed in 5 patients $(26.3 \%)$.

Table 1

Preoperative characteristics of patients who underwent endoscopic management of ureteral strictures $(n=19)$

\begin{tabular}{|ll|}
\hline Factor & $\mathrm{n}=19$ \\
\hline Sex & \\
\hline Male & $12(63.2 \%)$ \\
\hline Female & $7(36.8 \%)$ \\
\hline Age (years) ${ }^{\mathrm{a}}$ & $69[58,73]$ \\
\hline BMI $\left(\mathrm{kg} / \mathrm{m}^{2}\right)^{\mathrm{a}}$ & $24[21,26.9]$ \\
\hline Laterality & \\
\hline Right & $11(57.9 \%)$ \\
\hline Left & $8(42.1 \%)$ \\
\hline Location & \\
\hline Proximal & $7(36.8 \%)$ \\
\hline Distal & $12(63.2 \%)$ \\
\hline Cause of stricture & \\
\hline URSL & $10(52.6 \%)$ \\
\hline Impacted stone & $7(36.8 \%)$ \\
\hline chronic inflammatory disorders & $2(10.5 \%)$ \\
\hline Length of stricture & $10[7,19.5]$ \\
\hline Duration of hydronephrosis before surgery (months) & $4.0[2.5,5.5]$ \\
\hline Preoperative hydronephrosis & $5(26.3 \%)$ \\
\hline Grade 1 & $5(26.3 \%)$ \\
\hline Grade 2 & $6(31.6 \%)$ \\
\hline Grade 3 & \\
\hline Grade 4 & \\
\hline Prestenting & \\
\hline
\end{tabular}

BMI, body mass index; URSL, ureteroscopic lithotripsy 
${ }^{a}$ Median [25\%, 75\% interquartile range].

Surgical data are shown in Table 2. Eight patients (42.1\%) underwent both $\mathrm{LI}$ and BD. Two patients (10.6\%) underwent $\mathrm{LI}$ alone, and 3 patients (15.8\%) underwent BD alone. A single stent was placed in 8 patients (42.1\%) and double stents were placed in 9 patients (47.3\%). We could perform neither LI nor BD in 3 patients (15.8\%); therefore, we placed a ureteral stent in 1 patient $(5.3 \%)$ and a nephrostomy tube in 1 patient (5.3\%). Postoperative hydronephrosis was Grade 0 in 7 patients (36.8\%), Grade 1 in 3 patients (15.8\%), Grade 2 in 2 patients (10.5\%), Grade 3 in 4 patients (21.1\%), and Grade 4 in 3 patients (15.8\%). The success rate of the treatment was $63.2 \%$.

Table 2

Perioperative details of the endoscopic management of ureteral strictures

\begin{tabular}{|c|c|}
\hline Surgical time (min) a & $71.0[64.5,93.5]$ \\
\hline \multicolumn{2}{|l|}{ Endoscopic management } \\
\hline Lase incision (LI) and balloon dilation (BD) & $8(42.1 \%)$ \\
\hline LI & $2(10.6 \%)$ \\
\hline BD & $3(15.8 \%)$ \\
\hline \multicolumn{2}{|l|}{ Drainage } \\
\hline Single stent & $8(42.1 \%)$ \\
\hline Double stent & $9(47.3 \%)$ \\
\hline Nephrostomy tube & $1(5.3 \%)$ \\
\hline Duration of follow-up after surgery (months) ${ }^{a}$ & $8.0[3.0,17.0]$ \\
\hline \multicolumn{2}{|l|}{ Postoperative hydronephrosis } \\
\hline Grade 0 & $7(36.8 \%)$ \\
\hline Grade 1 & $3(15.8 \%)$ \\
\hline Grade 2 & $2(10.5 \%)$ \\
\hline Grade 3 & $4(21.1 \%)$ \\
\hline Grade 4 & $3(15.8 \%)$ \\
\hline Success of treatment & $12(63.2 \%)$ \\
\hline
\end{tabular}

$\mathrm{LI}$, laser incision; BD, balloon dilation

${ }^{a}$ Median [25\%, 75\% interquartile range]. 
Table 3 shows the data classified as failure or success of the treatment. URSL-related US occurred in 7 and 3 patients in the failure and success groups, respectively $(P=0.004)$. Contrarily, all seven patients with impacted stone-related US were successfully treated with endoscopic management. Five patients (71.4\%) had a US longer than $15 \mathrm{~mm}$ in the failure group, whereas two patients (16.6\%) had a US longer than $15 \mathrm{~mm}$ in the success group $(P=0.045)$. In all five patients who underwent presenting, endoscopic management was successful. The median duration of hydronephrosis before surgery was 5.0 months in the failure group and 4.0 months in the success group $(P=0.898)$. Both $L I$ and $B D$ were performed in four patients $(57.1 \%)$ in the failure group and in four patients $(33.3 \%)$ in the success group $(P=0.481)$. The median US lengths in the patients treated with both $\mathrm{LI}$ and $\mathrm{BD}$ in the failure and success groups were $22.5 \mathrm{~mm}$ and $10.0 \mathrm{~mm}$, respectively $(P=0.34)$. Double stents were placed in four patients $(57.1 \%)$ in the failure group and in five patients $(41.7 \%)$ in the success group $(P=0.12)$. The median US length in the patients who underwent double stent placement in the failure group was $22.5 \mathrm{~mm}$, whereas it was $10.0 \mathrm{~mm}$ in the success group $(P=0.34)$. 
Table 3

Comparison of factors associated with the success of endoscopic management for ureteral strictures

\begin{tabular}{|c|c|c|c|}
\hline Factor & Failure $(n=7)$ & $\begin{array}{l}\text { Success }(n= \\
12)\end{array}$ & $\begin{array}{l}P \\
\text { value }\end{array}$ \\
\hline Age (years) $^{a}$ & $\begin{array}{l}71.5[58.0, \\
75.3]\end{array}$ & $60.0[58.0,66.0]$ & 0.253 \\
\hline
\end{tabular}

Sex

Male

$3(42.9 \%)$

$9(75.0 \%)$

0.326

Female

$4(57.1 \%)$

$3(25.0 \%)$

$\operatorname{BMI}\left(\mathrm{kg} / \mathrm{m}^{2}\right)^{\mathrm{a}}$

$25.0[24.0$,

26.9]

$23.5[20.8,26.5]$

0.611

Cause of stricture

URSL

$7(100 \%)$

$3(25.0 \%)$

0.004

Impacted stone

$0(0.0 \%)$

$7(58.3 \%)$

chronic inflammatory disorders

$0(0.0 \%)$

$2(16.7 \%)$

Length of stricture $(>15 \mathrm{~mm}$ )

$5(71.4 \%)$

$2(16.6 \%)$

0.045

Prestenting

$0(0.0 \%)$

$5(41.7 \%)$

0.106

Duration of hydronephrosis before surgery (months)

$5.0[3.5,5.0]$

$4.0[2.0,7.5]$

0.898

a

Endoscopic management

Lase incision ( $\mathrm{LI})$ and balloon dilation (BD)

$4(57.1 \%)$

$4(33.3 \%)$

0.481

LI

$0(0.0 \%)$

$2(16.6 \%)$

$\mathrm{BD}$

$0(0.0 \%)$

$3(25.0 \%)$

Drainage

Single stent

$1(14.3 \%)$

$7(58.3 \%)$

0.12

Double stent

$4(57.1 \%)$

$5(41.7 \%)$

Nephrostomy tube

$1(14.3 \%)$

$0(0.0 \%)$

BMI, body mass index; URSL, ureteroscopic lithotripsy; LI, laser incision; BD, balloon dilation

${ }^{a}$ Median [25\%, 75\% interquartile range].

Table 4 outlines the causes of strictures. The success rate was $30.0 \%$ for URSL-related US, whereas it was $100 \%$ for impacted stone-related US $(P=0.01)$. Four patients $(40.0 \%)$ were male in the URSL-related US group, whereas seven patients $(100 \%)$ were male in the stone-related US group $(P=0.035)$. Seven patients 
(70.0\%) with URSL-related US had strictures longer than $15 \mathrm{~mm}$ in length compared to one patient $(14.3 \%)$ in the impacted stone-related US group $(P=0.049)$. Both $L I$ and BD were performed in five patients $(50.0 \%)$ in the URSL-related US group, whereas they were performed in two patients $(28.6 \%)$ in the impacted stone-related US group $(P=0.874)$. Double stents were placed in five patients $(50.0 \%)$ in the URSL-related US group and in five patients $(57.1 \%)$ in the impacted stone-related US group $(P=0.874)$.

Table 4

Comparison of factors associated with the cause of ureteral strictures

\begin{tabular}{|c|c|c|c|}
\hline Factor & URSL $(n=10)$ & impacted stone $(n=$ & $\begin{array}{l}P \\
\text { value }\end{array}$ \\
\hline Successfully treated cases & $3(30.0 \%)$ & $7(100 \%)$ & 0.01 \\
\hline Age (years) ${ }^{a}$ & $\begin{array}{l}61.5[57.0 \\
69.0]\end{array}$ & $75.0[67.5,77.5]$ & 0.063 \\
\hline \multicolumn{4}{|l|}{ Sex } \\
\hline Male & $4(40.0 \%)$ & $7(100 \%)$ & 0.035 \\
\hline Female & $6(60.0 \%)$ & $0(0.0 \%)$ & \\
\hline $\operatorname{BMI}\left(\mathrm{kg} / \mathrm{m}^{2}\right)^{\mathrm{a}}$ & $\begin{array}{l}24.5 \text { [21.8, } \\
27.3]\end{array}$ & $25.0[23.5,27.5]$ & 0.659 \\
\hline Length of stricture (> $15 \mathrm{~mm}$ ) & $7(70.0 \%)$ & $1(14.3 \%)$ & 0.049 \\
\hline Prestenting & $2(20.0 \%)$ & $2(28.6 \%)$ & 1 \\
\hline $\begin{array}{l}\text { Duration of hydronephrosis before surgery } \\
\text { (months) }{ }^{a}\end{array}$ & $4.5[3.3,5.0]$ & $4.0[2.5,8.0]$ & 0.693 \\
\hline \multicolumn{4}{|l|}{ Endoscopic management } \\
\hline Lase incision (LI) and balloon dilation (BD) & $5(50.0 \%)$ & $2(28.6 \%)$ & 0.874 \\
\hline LI & $0(0.0 \%)$ & $1(14.3 \%)$ & \\
\hline BD & $1(10.0 \%)$ & $1(14.3 \%)$ & \\
\hline \multicolumn{4}{|l|}{ Drainage } \\
\hline Single stent & $3(30.0 \%)$ & $3(42.9 \%)$ & 0.874 \\
\hline Double stent & $5(50.0 \%)$ & $5(57.1 \%)$ & \\
\hline Nephrostomy tube & $1(10.0 \%)$ & $0(0.0 \%)$ & \\
\hline \multicolumn{4}{|c|}{ URSL, ureteroscopic lithotripsy; BMI, body mass index; LI, laser incision; BD, balloon dilation } \\
\hline${ }^{a}$ Median $[25 \%, 75 \%$ interquartile range]. & & & \\
\hline
\end{tabular}


Renal function is shown in Table 5. The median preoperative and postoperative Creatinine (Cre) levels were 0.90 and 0.86 , respectively, and the median estimated glomerular filtration rates (eGFRs) were 60.6 and 60.9, respectively. In the patients with failed endoscopic management, the median preoperative and postoperative Cre were 0.89 and 0.82 , while the median eGFRs were 62.1 and 65.2 , respectively. In the patients with successful endoscopic management, the median preoperative and postoperative Cre were 0.96 and 0.98 , while the median eGFRs were 55.7 and 50.9 , respectively. The difference in the data reported in Table 5 are not significant.

Table 5

Overall preoperative and postoperative renal function and renal function after failed and successful endoscopic management

\begin{tabular}{|llll|}
\hline & Preoperation & Postoperation & P value \\
\hline Cre & $0.90[0.79,0.95]$ & $0.86[0.75,0.98]$ & 0.097 \\
\hline eGFR & $60.6[53.1,65.5]$ & $60.9[55.7,70.8]$ & 0.103 \\
\hline Failure & Preoperation & Postoperation & P value \\
\hline Cre & $0.89[0.83,0.93]$ & $0.82[0.72,0.93]$ & 0.399 \\
\hline eGFR & $62.1[59.8,73.8]$ & $65.2[60.3,73.0]$ & 0.563 \\
\hline Success & Preoperation & Postoperation & P value \\
\hline Cre & $0.96[0.78,1.14]$ & $0.98[0.82,1.19]$ & 0.23 \\
\hline eGFR & $55.7[44.4,58.8]$ & $50.9[45.9,57.2]$ & 0.154 \\
\hline Cre, creatinine; eGFR, estimated glomerular filtration rate \\
\hline \multicolumn{2}{|l}{ aMedian $[25 \%, 75 \%$ interquartile range $]}$. \\
\hline
\end{tabular}

\section{Discussion}

The development and innovation of endourologic tools has enabled urologists to choose endoscopic management techniques, such as endoureterotomy and endoscopic dilation, for patients with US. ${ }^{14}$ These techniques are safer and less invasive than open surgical repair; however, success rates vary widely between reports. ${ }^{9,15}$ We would investigate the factors which influence the success rate of the management for US.

Intraoperative ureteral damage during URSL is one of the causes of US. ${ }^{6}$ US caused by ureteral damage is associated with ischemic changes, which results in lower success rates following treatment for US ${ }^{7}$. On the other hand, it is reported that stones embedded in the ureteral mucosa stimulate inflammation, which might result in US. ${ }^{16}$ US caused by stones embedded in the ureteral wall does not always involve ischemic changes; therefore, it is likely to be curable with additional treatment. ${ }^{11}$ Netto et al. reported that 
the success rates of BD for non-ischemic and ischemic US were $89 \%$ and $29 \%$, respectively. ${ }^{17}$ In the current study, $52.6 \%$ of the USs were related to URSL; the success rate of the management for URSLrelated US was significantly lower compared to that of the management for impacted stone-related US ( $P$ $=0.01$ ). The US length was significantly longer in the patients with URSL-related US. The ischemic changes in the ureteral wall caused by laser lithotripsy may be associated with the development of a longer stricture. Together, these data suggest that to treat URSL-related US with endoscopic management is challenging.

US length is thought to be an important predictor of the outcome after endoscopic management for US in several reports. Netto et al. reported a lower success rate for the management of USs longer than $10 \mathrm{~mm} .{ }^{17}$ Thomas et al. reported a poorer outcome of BD for USs longer than $15 \mathrm{~mm} .{ }^{18}$ Meretyk et al. reported that the $20 \mathrm{~mm}$ in length was the most reliable predictor of success rate of $\mathrm{LI}^{15}$ The current study demonstrated that more than $70 \%$ of patients failed with endoscopic treatment had a US longer than $15 \mathrm{~mm}$. Consistent with previous reports, our study revealed that a length of $15 \mathrm{~mm}$ was likely to be an important factor to affect success rate of endoscopic treatment, even if the combination of LI and BD, as well as double stenting were utilized as novel techniques.

Prior studies report that the duration of the US is associated with the success rate for endoscopic management. Byun et al. reported that the duration of US (shorter or longer than 3 months) was an important factor which affected the success rate. ${ }^{19}$ On the other hand, Wolf et al. reported that the duration of the US did not significantly affect the success rate of US treatment. ${ }^{20}$ In the current study, the median duration of hydronephrosis before surgery was not significantly different between the patients with endoscopic treatment failure and success.

The success rate of $\mathrm{LI}$ using a holmium YAG laser was reported to be $67-68.4 \% .{ }^{21,22}$ Moreover, previous reports demonstrated that the success rate of BD for US was $50-76 \% .{ }^{23,24}$ A Holmium YAG laser with both cutting and coagulating functions provides precise incision to a depth of the fat tissue outside of the ureter with effective hemostatic effect. ${ }^{25}$ However, the laser incision or placement of a ureteral stent do not always sufficiently expand the lumen; therefore, we believe that the combination of LI and BD enables the equally centered expansion of the lumen on the incision line, which prevents restenosis. The current study showed that $57 \%$ patients underwent both $\mathrm{LI}$ and BD in the failure group; whereas one third of the patients underwent both procedures in the success group. There were no significant differences in the management between the patients with success or failure. However, the median length of US in the patients treated by both $\mathrm{LI}$ and $\mathrm{BD}$ in the success group of endoscopic management tended to be longer than that in the patients treated by both $\mathrm{LI}$ and $\mathrm{BD}$ in the failure group, although there was no significant difference. Thus, US length might affect the success rate of the combination of $\mathrm{LI}$ and BD as well.

Ureteral stents are preoperatively used for avoiding infection and kidney failure before the management of urolithiasis. They dilate the ureteral lumen and straighten the ureter, which makes it easy to insert a ureteroscope or ureteral access sheath. ${ }^{26}$ For these reasons, prestenting would also elevate the success 
rate of endoscopic management for US. In our study, all five patients with prestenting had successful endoscopic management.

The placement of two ureteral stents was first reported in cases of malignant obstruction. ${ }^{27}$ The authors suggested that two stents have more power to stand up to the comprehensive force of the tumor than one thick stent. The use of two ureteral stents has been applied for the management of benign US. 9,28,29 Some urologists prefer to insert as large of a ureteral stent as possible; however, larger stents cause ischemia of the ureter, which tend to develop restenosis. ${ }^{29}$ It is reported that two stents slide each other via peristalsis of the ureter, which maintain the expanded lumen. ${ }^{30}$ This motion may prevent ischemia or pressure necrosis of the ureter, which is believed to result in a better success rate. We found no statistical differences in treatment success rates between the patients with single and double stents. However, the median length of the US in patients in the success group who underwent double stent placement tended to be longer than in patients with double stents in the failure group, though there was no significant difference. Thus, the length of the US might affect the success rate.

Our study is limited by its relatively small number of patients. Moreover, it is difficult to assess which factor is most important for the success rate because we conducted different treatments for each patient. Furthermore, given that identifying the beginning of US development was difficult without close monitoring, we may not have been able to provide an accurate estimate of the US duration. Despite these limitations, we believe that our study findings are useful for improving the management of treatment for stone-related US.

\section{Conclusions}

In conclusion, we investigated the outcomes of endoscopic management for stone-related US at our institution. URSL as a cause and longer than $15 \mathrm{~mm}$ in length could strongly affect the success rate of the endoscopic management of US. In such cases, reconstructive management for US should be considered in the early stages.

\section{Abbreviations}

$\mathrm{BD}$, balloon dilation

BMI, body mass index

Cre, creatinine

eGFR, estimated glomerular filtration rate

LI, laser incision

URSL, ureteroscopic lithotripsy 
US, ureteral strictures

\section{Declarations}

\section{Ethics approval and consent to participate}

The present study was approved by the Institutional Review Board of Nagoya City University Hospital. All patients provided informed consent for the use of their data.

\section{Consent for publication}

All patients provided informed consent for the use of their data.

\section{Competing interests}

The authors declare that they have no competing interests.

\section{Availability of data and materials}

The datasets used analysed during the current study are available from the corresponding author on reasonable request.

\section{Funding}

Not applicable

\section{Authors' contributions}

TS: Manuscript writing and editing

KT: Project development and manuscript reviewing

SH: Data analyzing

Okada T: Data collection

MI: Data collection

YT: Data collection

RU: Data analyzing

YF: Data analyzing

TH: Statistical analysis

RA: Statistical analysis 
Okada A: Data management

TY: Manuscript reviewing and editing

\section{Acknowledgements}

Not applicable

\section{References}

1. Curhan GC. Epidemiology of stone disease. Urol Clin North Am. 2007;34:287-93.

2. Ando R, Nagaya T, Suzuki S, Takahashi H, Kawai M, Okada A, et al. Kidney stone formation is positively associated with conventional risk factors for coronary heart disease in Japanese men. $J$ Urol. 2013;189:1340-6.

3. Preminger GM, Tiselius HG, Assimos DG, Alken P, Buck C, Gallucci M, et al, EAU/AUA Nephrolithiasis Guideline Panel. 2007 Guideline for the management of ureteral calculi. J Urol. 2007;178:2418-34.

4. Taguchi K, Cho SY, Ng AC, Usawachintachit M, Tan YK, Deng YL, et al. The Urological Association of Asia clinical guideline for urinary stone disease. Int J Urol. 2019;26:688-709.

5. Geavlete P, Georgescu D, Nita G, Mirciulescu V, Cauni V. Complications of 2735 retrograde semirigid ureteroscopy procedures: A single-center experience. J Endourol. 2006;20:179-85.

6. Gdor Y, Gabr AH, Faerber GJ, Roberts WW, Wolf JS Jr. Success of laser endoureterotomy of ureteral strictures associated with ureteral stones is related to stone impaction. J Endourol. 2008;22:250711.

7. Roberts WW, Cadeddu JA, Micali S, Kavoussi LR, Moore RG. Ureteral stricture formation after removal of impacted calculi. J Urol. 1998;159:723-6.

8. Fam XI, Singam P, Ho CC, Sridharan R, Hod R, Bahadzor B, et al. Ureteral stricture formation after ureteroscope treatment of impacted calculi: a prospective study. Korean J Urol. 2015;56:63-7.

9. Razdan S, Silberstein IK, Bagley DH. Ureteroscopic endoureterotomy. BJU Int. 2005;95:94-101.

10. Ramanathan LT, Kumar A, Kapoor R, Bhandari M. Relief of urinary tract obstruction in tuberculosis to improve renal function: Analysis of predictive factors. Br J Urol. 1998;81:199-205.

11. Lang EK, Fritzsche PF. Ureteral strictures. In: Lang EK, editor. Radiology of the Lower Urinary Tract. Berlin: Springer-Verlag; 1994. pp. 33-40.

12. May PC, Hsi RS, Tran H, Stoller ML, Chew BH, Chi T, et al. The morbidity of ureteral strictures in patients with prior ureteroscopic stone surgery: multi-institutional outcomes. J Endourol. 2018;32:309-14.

13. Kanda Y. Investigation of the freely available easy-to-use software 'EZR' for medical statistics. Bone Marrow Transplant. 2013;48:452-8.

14. Richter F, Irwin RJ, Watson RA, Lang EK. Endourologic management of benign ureteral strictures with and without compromised vascular supply. Urology. 2000;55:652-7. 
15. Meretyk S, Albala DM, Clayman RV, Denstedt JD, Kavoussi LR. Endoureterotomy for treatment of ureteral strictures. J Urol. 1992;147:1502-6.

16. Dretler SP, Young RH. Stone granuloma: a cause of ureteral stricture. J Urol. 1993;150:1800-2.

17. Netto Júnior NR, Ferreira U, Lemos GC, Claro JF. Endourological management of ureteral strictures. J Urol. 1990;144:631-4.

18. Thomas R. Choosing the ideal candidate for ureteroscopic endoureterotomy. J Urol. 1993;149-314.

19. Byun SS, Kim JH, Oh SJ, Kim HH. Simple retrograde dilation for treatment of ureteral strictures: etiology-based analysis. Yonsei Med J. 2003;44:273-8.

20. Wolf JS, Soble JJ, Ratliff TL, Clayman RV. Ureteral cell cultures II. Collagen production and response to pharmacologic agents. J Urol. 1996;156:2067-72.

21. Singal RK, Denstedt JD, Razvi HA, Chun SS. Holmium: YAG laser endoureterotomy for treatment of ureteral stricture. Urology. 1997;50:875-80.

22. Lane BR, Desai MM, Hegarty NJ, Streem SB. Long-term efficacy of holmium laser endoureterotomy for benign ureteral strictures. Urology. 2006;67:894-7.

23. Schondorf D, Meierhans-Ruf S, Kiss B, Giannarini G, Thalmann GN, Studer UE, et al. Ureteroileal strictures after urinary diversion with an ileal segment-is there a place for endourological treatment at all? J Urol. 2013;190:585-90.

24. Laven BA, O'Connor RC, Gerber GS, Steinberg GD. Long-term results of endoureterotomy and open surgical revision for the management of ureteroenteric strictures after urinary diversion. $\mathrm{J}$ Urol. 2003;170:1226-30.

25. Johnson DE, Cromeens DM, Price RE. Transurethral incision of the prostate using the holmium:YAG laser. Lasers Surg Med. 1992;12:364-9.

26. Shields JM, Bird VG, Graves R, Gómez-Marín O. Impact of preoperative stenting on outcome of ureteroscopic treatment for urinary lithiasis. J Urol. 2009;182:2768-74.

27. Liu JS, Hrebinko RL. The use of 2 ipsilateral ureteral stents for relief of ureteral obstruction from extrinsic compression. J Urol. 1998;159:179-81.

28. Christman MS, Kalmus A, Casale P. Morbidity and efficacy of ureteroscopic stone treatment in patients with neurogenic bladder. J Urol. 2013;190:1479-83.

29. Ibrahim HM, Mohyelden K, Abdel-Bary A, Al-Kandari AM. Single versus double ureteral stent placement after laser endoureterotomy for the management of benign ureteral strictures: A randomized clinical trial. J Endourol. 2015;29:1204-9.

30. Isogai M, Hamamoto S, Hasebe K, lida K, Taguchi K, Ando R, et al. Dual ureteral stent placement after redo laser endoureterotomy to manage persistent ureteral stricture. IJU Case Rep. 2020;3:93-5. 